\title{
Sensibilização de profissionais de saúde para a redução de vulnerabilidades programáticas na hanseníase
}

Awareness among health professionals to reduce programmatic vulnerabilities in leprosy

Aderlene Rodrigues Pires*

\begin{abstract}
Resumo
O artigo discute os resultados de uma oficina pedagógica para sensibilização de profissionais de um Centro de Saúde Escola, unidade de referência na atenção à hanseníase, na região central de São Paulo. Realizou-se um estudo transversal, descritivo e quantitativo abrangendo o universo dos profissionais de nível médio (auxiliares de enfermagem e administrativos), participantes da oficina pedagógica conduzida com base na metodologia problematizadora de base sócio construtivista. Os dados foram coletados por meio da aplicação de um formulário anônimo no início e ao final do processo para aferir e comparar o nível de conhecimento dos profissionais sobre a hanseníase, posteriormente, submetidos à análise descritiva. Os resultados aferidos no pré-teste demonstraram desconhecimento sobre a doença e no pós-teste, evidenciaram que houve aumento no nível de informação dos profissionais na categoria "informação e conhecimento", no entanto, observa-se que o número de respostas corretas foi menor na categoria "medos, estigmas e preconceitos", indicando a continuidade dos processos de formação para redução das vulnerabilidades programáticas no acolhimento e suspeição dos casos de hanseníase.
\end{abstract}

Palavras-chave: Hanseníase. Capacitação em Serviço. Atenção Primária à Saúde. Populações Vulneráveis.

\begin{abstract}
The article discusses the results of an educational workshop aiming to raise awareness among health professionals in a Health Center School, leprosy's reference unit in the central area of São Paulo. A cross-sectional, descriptive and quantitative study covering the universe of the mid-level professionals (nursing and administrative assistants) was conducted, members of an educational workshop conducted based on the problem-solving methodology of a socio constructivist basis. Data were collected through an anonymous application form at the beginning and end of the process to assess and compare the level of knowledge of professionals about the disease, subsequently submitted to descriptive analysis. The results measured in the pretest showed a high degree of ignorance about the disease and the post-test showed that there was an increase in the level of information for professionals on the category "information and knowledge", however, it is observed that the number of correct responses was lower on the category "fears, stigmas and prejudices", indicating the continuity of training processes to reduce vulnerabilities in the host program and suspected cases of leprosy.
\end{abstract}

Keywords: Leprosy. In-service Training. Primary Health Care. Vulnerable Populations.

DOI: $10.15343 / 0104-7809.20153904484494$

* Centro de Saúde Escola Barra Funda - Dr. Alexandre Vranjac. São Paulo - SP, Brasil. E-mail: aderlenerpires@hotmail.com

** Instituto de Saúde da Secretaria de Estado da Saúde de São Paulo. São Paulo - SP, Brasil. E-mail: renato@isaude.sp.gov.br

Os autores declaram não haver conflitos de interesse. 


\section{INTRODUÇÃO}

A Organização Mundial da Saúde (OMS), baseada em informes epidemiológicos anuais indica que em 2013, o Brasil respondeu por 93,8\% das notificações de hanseníase nas Américas, ocupando a primeira posição no ranking de incidência. No cenário global, o país ocupa a segunda posição no ranking liderado pela Índia ${ }^{1}$.

A hanseníase é uma doença infecto-contagiosa, causada pelo Mycobacterium leprae, com tropismo pela pele e nervos periféricos, que pode conduzir ao dano neural, alteração de função de sensibilidade e/ou motora ${ }^{2}$. No cenário nacional nas últimas décadas, em que pese às mudanças induzidas pelas políticas públicas para a organização do cuidado e das práticas de saúde, a hanseníase ainda é considerada um grave problema de saúde pública, seja pelo seu potencial incapacitante ou por ser uma doença contagiosa ${ }^{2}$.

A OMS definiu dois planos estratégicos para implementação nos períodos de 2006 a 2010 e 2011 a 2015, com vistas a reduzir a carga da doença e promover o acesso ao diagnóstico precoce e o tratamento nos serviços de saúde ${ }^{3,4}$.

No Brasil, a hanseníase integra a lista de doenças negligenciadas, as quais constituem um conjunto de enfermidades de alta prevalência presentes no perfil sanitário dos países em desenvolvimento ${ }^{5}$. No âmbito do Sistema Único de Saúde (SUS), a implementação, o monitoramento e a avaliação das políticas e das ações programáticas, voltadas ao controle, à prevenção e ao tratamento da hanseníase são coordenadas pelo Ministério da Saúde em conjunto com as Secretariais Estaduais e Municipais. O Pacto pela Saúde publicado em 2006, vigente até o presente momento propõe o fortalecimento da resposta governamental no enfrentamento das doenças negligenciadas ${ }^{6}$. Assim, a meta de eliminação da hanseníase deverá ser alcançada pelo país até 2015, estimando-se um caso por 10.000 habitantes, o que implica envidar esforços para aprimorar a detecção precoce e a cura dos casos diagnosticados; a redução de incapacidades, do estigma e da discriminação, bem como a promoção da reabilitação social e econômica das pessoas afetadas ${ }^{6}$.

Diretrizes publicadas pelo Ministério da Saúde em 2000 e 2009, nas portarias № 816 e $N^{\circ} 125$, respectivamente, alertam que a eliminação da hanseníase depende de outros fatores, como a redução da morbidade e dos danos decorrentes da gravidade das incapacidades físicas, psíquicas e sociais. Nessa perspectiva, o atendimento à demanda espontânea com prioridade para os pacientes sintomáticos dermato-neurológicos, a busca ativa dos comunicantes e as ações de educação em saúde junto à população são elementos fundamentais que devem ser observados pelas equipes na organização da atenção e do processo de trabalho na rede de atenção básica ${ }^{6,7,8}$.

Fernandes e Telles Filho ao analisarem a trajetória da hanseníase em diversas regiões do Brasil destacam avanços no quadro sanitário, como o conhecimento do agente etiológico e as possibilidades de cura da doença. No entanto, apontam como desafio primordial, a superação das situações de medo, preconceito e segregação que afetam a pessoa e sua família e aumentam as vulnerabilidades, sobretudo na dimensão individual e social ${ }^{9,10}$.

Investir na qualificação das tecnologias que envolvem o processo de acolhimento nos serviços de saúde também é essencial para minimizar a vulnerabilidade programática no que se refere à complexidade do diagnóstico e do tratamento da hanseníase ${ }^{10}$. O acolhimento pressupõe mudanças significativas na organização do processo de trabalho da equipe, baseados na garantia da acessibilidade universal; na integralidade da atenção multiprofissional; e na humanização dos vínculos e das relações estabelecidas no lócus dos serviços de saúde ${ }^{11}$.

Nessa ótica, o acolhimento conduzido pela equipe multiprofissional é fundamental para o diagnóstico precoce e à adesão aos protocolos definidos no plano terapêutico, associado às ações de prevenção secundária para a redução do risco de incapacidades, sequelas e deformidades e o aumento da autopercepção das pessoas afetadas pela doença ${ }^{12}$. 
O Ministério da Saúde tem envidado esforços para promover a qualificação da força de trabalho no SUS, em especial, na atenção primária à saúde, onde está em curso um processo de reorientação das estratégias e dos modos de cuidar nas dimensões individual e coletiva. Essas mudanças também afetam as estratégias téorico-metodológicas adotadas nos processos de formação e de ensino-aprendizagem no cotidiano dos serviços ${ }^{13}$.

Assim, um dos objetivos centrais da força de trabalho é produzir um contingente maior de trabalhadores tecnicamente qualificados e com atributos sociais e humanos adequados para lidar com as necessidades de saúde dos diferentes segmentos da população, bem como para planejar e incorporar novas práticas e, assumir responsabilidades compatíveis com o papel profissional na equipe ${ }^{14}$.

Cabe ainda contextualizar que os avanços tecnológicos e as mudanças recentes no perfil da distribuição da renda e da escolaridade da população brasileira, exigem uma força de trabalho mais especializada. Nesse sentido, o investimento na formação e educação permanente dos trabalhadores, associado às avaliações de processo e resultado das metodologias pedagógicas adotadas são elementos essenciais para intervir e reduzir a vulnerabilidade programática. Assim, a formação e o desenvolvimento profissional, por meio do diálogo, da reflexão e da problematização são estratégias importantes para alinhar as práticas de saúde à integralidade da atenção no SUS ${ }^{15}$.

O presente artigo tem por objetivo descrever o grau de conhecimento sobre a hanseníase após a realização de atividade educativa com profissionais de um Centro de Saúde Escola na cidade de São Paulo.

\section{METODOLOGIA}

Trata-se de um estudo transversal, descritivo e quantitativo. $\mathrm{O}$ estudo foi realizado em uma unidade de referência para atenção à hanseníase localizada na região centro-oeste do município de São Paulo. Esse serviço atua na atenção primária à saúde e desenvolve ações de ensino e pesquisa, contando com três equipes de Saúde da família (ESF) e uma equipe "especial-rua".

A população do território é heterogênea e desde 1995 a unidade desenvolve novas tecnologias para a atenção primária junto aos segmentos mais vulneráveis e em situação de exclusão social, como pessoas em situação de rua, moradores de cortiços e pequenos núcleos de favelas, trabalhadores do sexo, travestis, ambulantes e imigrantes ilegais, principalmente bolivianos ${ }^{16}$.

O Centro de Saúde Escola registrou entre 2008 e 2013 sete casos de hanseníase ${ }^{17}$, evidenciando fragilidades na condução das ações de suspeição da doença pelos profissionais. A ausência de capacitação técnica dos profissionais de nível médio pode ser uma dessas causas e revela um grau de vulnerabilidade programática a ser enfrentado.

Incluíram-se no estudo todos os profissionais que desempenhavam a função de auxiliar de enfermagem (19) e auxiliar administrativo (14), perfazendo um total de 36 trabalhadores envolvidos nas ações de acolhimento e suspeição dos casos de hanseníase. Excluíram-se os profissionais que estavam em licença médica ou em plantão na unidade na data de realização da atividade educativa.

Realizou-se uma oficina pedagógica com duração de quatro horas em julho de 2011, a fim de sensibilizar os profissionais sobre a problemática da hanseníase e definir um novo fluxo para atenção aos usuários. A oficina foi conduzida pela enfermeira coordenadora do serviço de vigilância epidemiológica. Para o desenvolvimento do conteúdo programático, adotou-se a metodologia problematizadora de base construtivista, abordando-se por meio de técnicas dialógicas, a construção e a reconstrução de conceitos técnico-científicos sobre a hanseníase, a importância das tecnologias de acolhimento e a redefinição do fluxo para organização do processo de trabalho da equipe.

Para a coleta dos dados aplicou-se um formulário anônimo no início e ao final da oficina com o objetivo de aferir o nível de conhecimento dos profissionais sobre a hanseníase. Os instrumentos do pré e pós-teste foram aplicados 
após esclarecimentos da enfermeira sobre os objetivos da proposta e a concordância dos participantes. Os dados foram organizados em planilha Excel e submetidos à análise descritiva baseada em frequências absolutas e relativas.

O formulário de coleta constou de 11 afirmações, autoavaliadas pelos profissionais, segundo a escala: (C) concordo totalmente; (D) discordo totalmente; e (NS) não sei a resposta correta. Informações adicionais, como a idade, o tempo de serviço em anos na área da saúde e a categoria profissional também foram incluídas para caracterização dos participantes. Na mensuração dos dados e totalização das frequências não foram consideradas as questões não respondidas. As questões foram agrupadas em três categorias:

Categoria 1: informação e conhecimento sobre a doença. Este grupo de questões versou sobre os conhecimentos em hanseníase, as formas de contágio, os sintomas, a cura e a importância do tratamento, abordando-se:

Q1. Hanseníase, lepra, moléstia de hansen são sinônimos.

Q2. Hanseníase é uma doença infecciosa, com longo período de incubação, causada por um bacilo.

Q4. Formigamento, perda de sensibilidade, perda de pêlos e dor são sinais de hanseníase.

Q7. Existem quatro tipos diferentes de hanseníase.

Q8. Se não tratada precocemente a hanseníase provoca deformidades.

Nessas afirmações, as respostas (C) foram consideradas adequadas e indicaram presença de conhecimento e as respostas (D) ou (NS) como falta de conhecimento.

Q3. A pessoa com hanseníase nunca se cura.

A resposta (D) foi considerada como presença de conhecimento e as respostas (C) ou (NS) como falta de conhecimento.

Categoria 2: Organização do serviço e fluxo. Foram apresentadas duas questões sobre a organização do processo de trabalho da equipe e a organização do fluxo de acolhimento aos usuários para suspeição da hanseníase, abordando-se:
Q5. Suspeitos de hanseníase devem ser agendados com o clínico geral.

A resposta (D) foi considerada como presença de conhecimento e as respostas (C) ou (NS) como falta de conhecimento.

Q11. Os retornos devem ser agendados a cada 28 dias.

A resposta $(\mathrm{C})$ foi considerada como presença de conhecimento e as respostas (D) e (NS) como falta de conhecimento.

Categoria 3: Medos, estigmas e preconceitos. Estas três afirmações expressaram situações e representações sociais dos trabalhadores associadas a possíveis medos e preconceitos relacionados à hanseníase, abordando-se:

Q6. Quando pensamos em hanseníase as primeiras coisas que vem à mente são deformidades e incapacidades.

Q9. Durante os quinze primeiros dias de tratamento o paciente deve ser afastado do trabalho.

Q10. O paciente deve ser orientado a dormir separado de seus familiares e a separar garfos, facas, copos, roupas pessoais, etc.

As respostas (D) foram consideradas como ausência de medos e preconceitos e as respostas (C) ou (NS) como presença de medos e preconceitos.

\section{RESULTADOS E DISCUSSÃO}

No Brasil, a hanseníase é uma doença negligenciada, no entanto, qualquer profissional da saúde devidamente capacitado pode realizar o diagnóstico precoce e a suspeição da doença, contribuindo para a redução da incidência e o avanço da prevenção de danos e incapacidades físicas, psíquicas e sociais ${ }^{2,5}$. Nessa ótica, as estratégias de educação permanente dirigidas aos profissionais da saúde devem ser desenvolvidas nas unidades da atenção básica para qualificar a integralidade dos cuidados à população e fortalecer as práticas de saúde em curso no SUS, em consonância com as diretrizes do Pacto pela Saúde ${ }^{6}$.

No presente estudo, a adesão dos trabalhadores a oficina foi considerada alta, perfazendo um total de 33 profissionais (92\%) dos 36 convidados para essa atividade educativa. $\mathrm{Na}$ 
ocasião, uma funcionária estava em licença médica e outros dois em atendimento no plantão da unidade. O universo de participantes foi constituído por 19 auxiliares de enfermagem e 14 auxiliares administrativos.

A Tabela 1 demonstra que os profissionais na faixa entre 20 e 30 anos (39,4\%) foram os mais frequentes na oficina, seguido dos trabalhadoperfil etário, majoritariamente, constituído por faixa etária, São Paulo - SP, julho 2011.
A Tabela 2 indica que na ocasião da oficina, a maioria dos participantes, possuía até cinco anos de atividade profissional em serviços de saúde $(36,4 \%)$, seguido pelos profissionais na faixa entre 6 e 10 anos (27,3\%). O menor tempo tempo de serviço em anos, São Paulo - SP, julho 2011. jovens adultos, condizente com a característica desse Centro de Saúde Escola, o qual conta com a presença de muitos alunos e residentes de Medicina. Os participantes com 50 anos ou mais responderam por $15,1 \%$ dos treinandos e dentre esses, os auxiliares administrativos $(21,4 \%)$ representaram o dobro dos trabalhadores comparados aos auxiliares de enfermagem $(10,5 \%)$. O profissional mais jovem tinha 20 anos e o mais idoso 69 anos.

Tabela 1. Frequência absoluta e relativa dos participantes da oficina, segundo categoria profissional e

\begin{tabular}{lcccccc} 
Faixa etária & $\begin{array}{c}\text { Aux. enf. } \\
\mathbf{N}^{\mathbf{0}}\end{array}$ & $\%$ & $\begin{array}{c}\text { Aux. adm. } \\
\mathbf{N}^{\mathbf{0}}\end{array}$ & $\%$ & $\begin{array}{c}\text { Total } \\
\mathbf{N}^{\mathbf{0}}\end{array}$ & $\%$ \\
\hline 20 a 30 & 5 & 26,3 & 8 & 57,2 & 13 & 39,4 \\
31 a 40 & 6 & 31,6 & 1 & 7,1 & 7 & 21,3 \\
41 a 50 & 5 & 26,3 & 0 & 0,0 & 5 & 15,1 \\
$>50$ & 2 & 10,5 & 3 & 21,4 & 5 & 15,1 \\
Sem inf. & 1 & 5,3 & 2 & 14,3 & 3 & 9,1 \\
\hline Total & $\mathbf{1 9}$ & $\mathbf{1 0 0}$ & $\mathbf{1 4}$ & $\mathbf{1 0 0}$ & $\mathbf{3 3}$ & $\mathbf{1 0 0}$ \\
\hline
\end{tabular}

de trabalho aferido foi de um mês e o maior de 32 anos. Na comparação entre as duas funções, verificou-se que, não houve diferença entre as mesmas quanto ao tempo dedicado ao exercício laboral na área da saúde pública.

Tabela 2. Frequência absoluta e relativa dos participantes da oficina, segundo categoria profissional e

\begin{tabular}{lcccccc}
\hline Tempo & $\begin{array}{c}\text { Aux. enf. } \\
\mathbf{N}^{\mathbf{0}}\end{array}$ & $\mathbf{\%}$ & $\begin{array}{c}\text { Aux. adm. } \\
\mathbf{N}^{\mathbf{0}}\end{array}$ & $\%$ & $\begin{array}{c}\text { Total } \\
\mathbf{N}^{\mathbf{0}}\end{array}$ & $\%$ \\
\hline$<1$ & 2 & 10,5 & 2 & 14,3 & 4 & 12,1 \\
1 a 5 & 7 & 36,8 & 5 & 35,7 & 12 & 36,4 \\
6 a 10 & 6 & 31,6 & 3 & 21,4 & 9 & 27,3 \\
10 ou + & 4 & 21,1 & 4 & 28,6 & 8 & 24,2 \\
\hline Total & $\mathbf{1 9}$ & $\mathbf{1 0 0}$ & $\mathbf{1 4}$ & $\mathbf{1 0 0}$ & $\mathbf{3 3}$ & $\mathbf{1 0 0}$ \\
\hline
\end{tabular}

As respostas corretas do pré-teste, aferidas na categoria "informação e conhecimento sobre a doença" foram superiores a 75\%, exceto quanto à questão que abordou a possibilidade de deformidades em decorrência do não tratamento da hanseníase, perfazendo apenas 40,6\% dos acertos (Tabela 3). Na categoria "organização do serviço/fluxo" a maioria desconhecia que os casos suspeitos de hanseníase devem ser agendados com o clínico geral da unidade; e pouco 
mais da metade referiu a periodicidade mensal dos retornos ao serviço. O grau de assertividade dos treinandos nessas duas categorias revelou o desconhecimento de uma parcela dos trabaIhadores em relação aos aspectos básicos da doença, evidenciando vulnerabilidades programáticas, oriundas da formação dos profissionais e que podem dificultar a organização das atividades de orientação e acolhimento pela equipe do serviço, diminuindo assim, a efetividade dos protocolos definidos pelo Ministério da Saúde para o diagnóstico precoce, o tratamento e a cura da doença ${ }^{8,9,10 .}$

No pré-teste, a categoria "medos, estigmas e preconceitos" apresentou o menor percentual de acertos, inferior a 58\%. A maioria dos profissionais associou a doença com deformidades e incapacidades, revelando representações sociais baseadas em preconceitos e no senso comum; e que é necessário o afastamento das atividades laborais no inicio do tratamento. Pouco mais da metade dos treinandos, responderam corretamente que não se deve orientar as pessoas com hanseníase a dormirem separado dos familiares ou compartilharem objetos de uso comum no lar.

Nos serviços de saúde, o enfrentamento dos medos e preconceitos relacionados à doença implica investir no desenvolvimento de processos educativos, com vistas à promoção das habilidades e das competências técnicas da força de trabalho para a efetividade das ações ${ }^{18}$. Vale sublinhar que as diretrizes do Ministério da Saúde, incentivam a educação permanente dos profissionais e alertam que, somente a transmissão da informação técnica, dissociada de processos pedagógicos coletivos baseados na problematização e na desmistificação dos medos e preconceitos, não é suficiente para o enfrentamento dessa questão no cotidiano das equipes ${ }^{12,13}$.

Tabela 3. Frequência absoluta e relativa das questões respondidas corretamente no pré-teste, São Paulo - SP, julho 2011.

\begin{tabular}{lll}
\hline Questão & $\mathbf{N}^{\mathbf{0}}$ & $\%$ \\
\hline Conhecimento sobre a doença & & \\
\hline Hanseníase, Lepra e Hansen são sinônimos & 28 & 87,5 \\
Tem incubação longa, causada por um bacilo & 26 & 81,3 \\
Hanseníase não tem cura & 26 & 78,8 \\
Principais sinais: formigamento e perda de sensibilidade & 26 & 81,3 \\
Existem 4 tipos de Hanseníase & 25 & 75,8 \\
Provoca deformidades se não tratada & 13 & 40,6 \\
\hline Organização do serviço/fluxo & 11 & 35,5 \\
\hline Agendar casos suspeitos com clínico geral & 21 & 63,6 \\
Agendar retornos a cada 28 dias & & \\
\hline Medos, estigmas e preconceitos & 14 & 42,4 \\
\hline Não associa a doença com deformidades e incapacidades & 11 & 33,3 \\
Não necessita afastamento do trabalho & 19 & 57,6 \\
\hline Não se orienta dormir e separar objetos de uso comum & & \\
\hline
\end{tabular}

Na Tabela 4, observam-se diferenças no grau de conhecimento dos treinandos, segundo as funções desempenhadas na unidade de saúde.
Constatou-se que os auxiliares de enfermagem foram mais assertivos nas categorias "organização do serviço/fluxo" e "medos, estigmas 
e preconceitos". Nessas categorias, o menor nível de informação foi aferido entre os auxiliares administrativos, revelando fragilidades no desempenho da organização do processo de trabalho da unidade, onde esses profissionais atuam na recepção, na orientação e no agendamento das consultas dos usuários com suspeita de hanseníase. No entanto, o percentual de acertos dos dois grupos foi menor do que $45 \%$, quando perguntados sobre a possibilidade de deformidades em decorrência do não tratamento da doença, novamente inferior entre os auxiliares administrativos, os quais alcançaram apenas $35,7 \%$ de acertos. categoria profissional, São Paulo - SP, julho 2011.
Cabe ponderar que, no âmbito da saúde pública, os aspectos associados ao conhecimento técnico da hanseníase são considerados obrigatórios na formação curricular dos profissionais de nível médio da enfermagem, almejando-se, portanto, uma maior qualificação no desempenho desses trabalhadores. Esse cenário reitera a relevância das ações pedagógicas para o aprimoramento das condutas e das rotinas adotadas no serviço de saúde, lócus em que esses trabalhadores são reconhecidos pela equipe multiprofissional como uma categoria-chave para a efetividade do acolhimento aos usuários.

Tabela 4. Frequência absoluta e relativa das questões respondidas corretamente no pré-teste, segundo

\begin{tabular}{lcccc}
\hline \multirow{2}{*}{ Questão } & \multicolumn{2}{c}{ Aux. enf. } & \multicolumn{2}{c}{ Aux. adm. } \\
\cline { 2 - 5 } & No & $\%$ & No & \% \\
\hline Conhecimento sobre a doença & & & & \\
\hline Hanseníase, Lepra e Hansen são sinônimos & 18 & 100 & 10 & 71,4 \\
Tem incubação longa, causada por um bacilo & 14 & 77,8 & 12 & 85,7 \\
Hanseníase não tem cura & 17 & 89,5 & 9 & 64,3 \\
Principais sinais: formigamento e perda de sensibilidade & 14 & 73,7 & 12 & 92,3 \\
Existem 4 tipos de Hanseníase & 14 & 73,7 & 11 & 78,6 \\
Provoca deformidades se não tratada & 8 & 44,4 & 5 & 35,7 \\
\hline Organização do serviço/fluxo & & & & \\
\hline Agendar casos suspeitos com clínico geral & 9 & 52,9 & 2 & 14,3 \\
Agendar retornos a cada 28 dias & 16 & 84,2 & 5 & 35,7 \\
\hline Medos, estigmas e preconceitos & & & & \\
\hline Não associa a doença com deformidades e incapacidades & 9 & 47,4 & 5 & 35,7 \\
Não necessita afastamento do trabalho & 10 & 52,6 & 1 & 7,1 \\
Não se orienta dormir e separar objetos de uso comum & 14 & 73,7 & 5 & 35,7 \\
\hline
\end{tabular}

Os resultados do pós-teste (Tabela 5), demonstraram aumento do nível de informação dos profissionais, perfazendo $100 \%$ de assertividade em todas as questões da categoria "informação e conhecimento sobre a doença". Quanto à "organização do serviço/fluxo" também houve incremento no conhecimento dos trabalhadores, sobretudo no que tange a periodicidade do agendamento dos retornos para o tratamento dos casos de hanseníase, de $63,6 \%$ no pré-teste para 93,3\%; e na marcação de consultas com o clínico geral, constatando-se 67,9\% de acertos, contra 35,5\% do pré-teste. Quanto à categoria "medos, estigmas e preconceitos" observou-se que o número de respostas corretas foi menor em relação às anteriores. Verificou-se que hou- 
ve progresso no conhecimento dos treinandos, somente na questão que abordou a orientação adequada do paciente quanto à convivência no espaço familiar e doméstico, aumentando de $57,6 \%$ para $96,7 \%$. Contudo, no plano das representações sociais, a metade dos profissionais continuou associando a doença a deformidades e incapacidades (de $42,4 \%$ para $50 \%$ ); e não houve mudança na opinião dos mesmos quanto à necessidade de afastamento do trabalho para o tratamento na primeira quinzena, permanecendo em $33,3 \%$.

Tabela 5. Frequência absoluta e relativa das questões respondidas corretamente no pós-teste, São Paulo - SP, julho 2011.

\begin{tabular}{|c|c|c|}
\hline Questão & $\mathbf{N}^{0}$ & $\%$ \\
\hline \multicolumn{3}{|l|}{ Conhecimento sobre a doença } \\
\hline Hanseníase, Lepra e Hansen são sinônimos & 30 & 100 \\
\hline Tem incubação longa, causada por um bacilo & 30 & 100 \\
\hline Hanseníase não tem cura & 30 & 100 \\
\hline Principais sinais: formigamento e perda de sensibilidade & 30 & 100 \\
\hline Existem 4 tipos de Hanseníase & 30 & 100 \\
\hline Provoca deformidades se não tratada & 30 & 100 \\
\hline \multicolumn{3}{|l|}{ Organização do serviço/fluxo } \\
\hline Agendar casos suspeitos com clínico geral & 19 & 67,9 \\
\hline Agendar retornos a cada 28 dias & 28 & 93,3 \\
\hline \multicolumn{3}{|l|}{ Medos, estigmas e preconceitos } \\
\hline Não associa a doença com deformidades e incapacidades & 15 & 50 \\
\hline Não necessita afastamento do trabalho & 10 & 33,3 \\
\hline Não se orienta dormir e separar objetos de uso comum & 29 & 96,7 \\
\hline
\end{tabular}

No pós-teste (Tabela 6), a análise revelou que a categoria "informação e conhecimento sobre a doença" não apresentou diferenças entre os trabalhadores. Observou-se um incremento considerável no nível de informação dos auxiliares administrativos, o qual passou de $14,3 \%$ no pré-teste para $70 \%$ de acertos no pós-teste, quanto ao agendamento adequado dos casos suspeitos; e nos retornos mensais ao serviço de $35,7 \%$ para $91 \%$. Entre os auxiliares de enfermagem, o agendamento com o clínico teve um pequeno aumento de 52,9\% para $66,7 \%$, mas aquém do preconizado nas diretrizes técnicas ${ }^{2,7,9}$. Quanto aos medos e estigmas presentes nas representações sociais da doença, constatou-se aumento na informação dos grupos sobre a orientação adequada no âmbito do con- vívio doméstico, entretanto, não foram alcançadas as mudanças esperadas no conhecimento dos profissionais sobre a associação da doença com deformidades e incapacidades, questão que ficou abaixo de $58 \%$ entre os auxiliares de enfermagem e $37 \%$ entre os administrativos. Não obstante os avanços obtidos no pós-teste, essas evidências reforçam a necessidade da continuidade dos processos educativos para o aperfeiçoamento dos fluxos estabelecidos, com vistas à efetividade dos protocolos terapêuticos definidos pelo Ministério da Saúde para o controle da hanseníase ${ }^{2,12}$.

Nessa perspectiva, estudos indicam que diversos fatores condicionam o acesso e o uso dos serviços de saúde pela população, destacando-se a qualidade do cuidado oferecido pelas equipes 
multiprofissionais ${ }^{14}$. O enfrentamento da vulnerabilidade programática na atenção à hanseníase nas unidades da atenção básica implica desenvolver processos proativos de formação e atualização técnica, qualificando o planejamento, a implementação e a avaliação contínua das práticas de saúde, em especial as tecnologias de acolhimento e aconselhamento voltadas ao diagnóstico precoce e ao tratamento eficaz para a quebra da cadeia de transmissão da doença $\mathrm{a}^{19}$.

Tabela 6. Frequência absoluta e relativa das questões respondidas corretamente no pós-teste, segundo categoria profissional, São Paulo - SP, julho 2011.

\begin{tabular}{lcccc}
\hline \multirow{2}{*}{ Questão } & \multicolumn{2}{c}{ Aux. enf. } & \multicolumn{2}{c}{ Aux. adm. } \\
\cline { 2 - 5 } & No & $\%$ & $\mathbf{N}^{\mathbf{0}}$ & $\%$ \\
\hline Conhecimento sobre a doença & & & & \\
\hline Tem incubação longa, causada por um bacilo & 19 & 100 & 11 & 100 \\
Hanseníase não tem cura & 19 & 100 & 11 & 100 \\
Principais sinais: formigamento e perda de sensibilidade & 19 & 100 & 11 & 100 \\
Existem 4 tipos de Hanseníase & 19 & 100 & 11 & 100 \\
Provoca deformidades se não tratada & 19 & 100 & 11 & 100 \\
\hline Organização do serviço/fluxo & & & & \\
\hline Agendar casos suspeitos com clínico geral & 12 & 66,7 & 7 & 70 \\
Agendar retornos a cada 28 dias & 19 & 100 & 10 & 91 \\
\hline Medos, estigmas e preconceitos & & & & \\
\hline Não associa a doença com deformidades e incapacidades & 11 & 57,9 & 4 & 36,4 \\
Não necessita afastamento do trabalho & 11 & 36,7 & 11 & 100 \\
Não se orienta dormir e separar objetos de uso comum & 19 & 100 & 10 & 90,9 \\
\hline
\end{tabular}

Assim, para reduzir a vulnerabilidade programática é fundamental que as equipes observem alguns aspectos que integram a atenção primária à saúde, elencados por Starfield: o primeiro contato (porta de entrada), responsável pela acessibilidade e o uso dos serviços; a longitudinalidade do cuidado, baseada na garantia da oferta regular da atenção ao longo do tempo, valorizando-se as relações e os vínculos entre o serviço e os usuários; a integralidade da atenção em consonância às necessidades de saúde da população adscrita; e a garantia da atenção nos níveis mais complexos quando necessário ${ }^{20}$.

Cabe ressaltar que, o acesso e o acolhimento dos usuários dependem da capacidade de reconhecimento das suas necessidades de saúde, elemento essencial na produção do cuidado. Assim, é mister qualificar todos os trabalha- dores da equipe, incluindo os de nível médio, corresponsáveis pelo processo de vinculação dos usuários ao serviço e pela integralidade da atenção preconizada no SUS, fortalecendo a longitudinalidade do cuidado na rede de saúde. Nesse aspecto, concordamos com Peduzzi ao ponderar que "à medida que o trabalhador domina o conteúdo do seu trabalho, amplia a sua esfera de autonomia criando espaço para que possa discutir, argumentar, reivindicar e interferir nas decisões do processo de trabalho" (p. 122) $)^{15}$. Portanto, faz-se necessário assegurar o envolvimento dos trabalhadores de nível médio, sobretudo os administrativos, os quais ainda são pouco convidados a participarem de processos de formação nas unidades da atenção básica.

Do ponto de vista técnico, também é importante que essas categorias profissionais estejam 
preparadas para escutar as pessoas com hanseníase, quanto as suas percepções, medos e dúvidas sobre a doença no que tange ao cotidiano social e familiar, orientando-as corretamente sobre a relevância da adesão e a continuidade do tratamento, bem como para o enfrentamento da discriminação e do preconceito, minimizando vulnerabilidades decorrentes do processo de estigmatização social das pessoas acometidas pela hanseníase ${ }^{9,21}$.

Os achados do presente estudo, especialmente em relação à categoria "medos, estigmas e preconceitos", demonstraram a complexidade dos processos educativos e os desafios que devem ser trilhados para desconstruir e modificar as representações sociais negativas dos trabalhadores sobre a hanseníase. Alguns autores chamam a atenção para as limitações das ações de capacitação na produção de mudanças nas condutas profissionais ${ }^{15}$. Contudo, as diretrizes da educação permanente preconizam o desenvolvimento de processos coletivos de ensino-aprendizagem no lócus institucional, com vistas à ressignificação da dinâmica do trabalho em equipe, proporcionando uma ferramenta com potência para transformar as práticas de saúde e a própria organização do processo laboral ${ }^{13}$.

Nessa ótica, a rede de atenção básica em consonância com as diretrizes do Pacto pela Saúde, possui um papel estratégico para o aumento da cobertura das ações voltadas ao diagnóstico precoce e ao autocuidado para evitar complicações e sequelas e, a melhoria da qualidade de vida das pessoas acometidas pela hanseníase ${ }^{6}$. Nesse espaço, as ações de educação em saúde para o controle da doença, desenvolvidas por todos os membros da equipe, incluindo os trabalhadores de nível médio, são essenciais para o incremento das tecnologias de prevenção primária e secundária no âmbito do território, fortalecendo as estratégias de promoção da saúde e do cuidado adotadas ${ }^{21}$.

Não obstante aos desafios elencados anteriormente, cabe reiterar a relevância do desen- volvimento das atividades educativas sobre a problemática da hanseníase, voltadas aos profissionais da saúde de nível médio, qualificando o desempenho da sua força de trabalho no âmbito da equipe multiprofissional.

\section{CONCLUSÃO}

A metodologia problematizadora de base sócio construtivista adotada na oficina pedagógica sensibilizou e aumentou o grau de conhecimento dos profissionais de nível médio do Centro de Saúde Escola, no que se refere à importância da orientação e do acolhimento dos usuários para o diagnóstico precoce e o tratamento oportuno da hanseníase. Contudo, as evidências da análise comparada dos resultados do pré e do pós-teste, demonstram que ainda há lacunas no nível de conhecimento dos profissionais, as quais devem ser objeto de continuidade das ações de formação, com vistas ao aprimoramento do processo de trabalho da equipe multiprofissional, sobretudo entre os trabalhadores que desempenham funções administrativas.

O principal desafio do processo de formação refere-se às mudanças nas representações sociais negativas dos trabalhadores que ainda reproduzem o estigma social da doença e podem dificultar a efetividade das orientações e dos protocolos terapêuticos. Assim, a redução da vulnerabilidade programática na atenção à hanseníase no âmbito dos serviços da atenção primária à saúde depende de investimentos dos gestores e das equipes locais em processos que qualifiquem a força de trabalho, ancorados nos pressupostos da educação permanente em saúde no Sistema Único de Saúde.

Por fim, baseado nos resultados desse estudo, cabe recomendar o desenvolvimento de outras pesquisas que aprofundem as discussões sobre a formação e a inserção dos profissionais de nível médio das categorias de enfermagem e administrativa no âmbito da atenção primária à saúde.

\section{REFERÊNCIAS}

1. World Health Organization. Global Leprosy Situation. Weekly Epidemiological Record. 5 septembre 2014; 36(89): $389-400$. 
2. Brasil. Ministério da Saúde. Departamento de Atenção Básica. Guia para controle de hanseníase. Brasília: Ministério da Saúde; 2002.

3. Organização Mundial da Saúde. Estratégia Global para aliviar a carga da Hanseníase e manter as atividades de controle da Hanseníase. Plano 2006-2010. Genebra; 2005.

4. Organização Mundial da Saúde. Estratégia global aprimorada para redução adicional da carga da hanseníase 2011-2015: diretrizes operacionais atualizadas. Brasilia: Organização Pan-Americana da Saúde; 2010.

5. Villa TCS. Estratégias de pesquisa para o controle de doenças negligenciadas: projetos colaborativos de enfermagem em rede. Rev. Latino-Am. Enfermagem. 2009; 17(4):437-8.

6. Brasil. Ministério da Saúde. Portaria GM n. 399, de 22 de fevereiro de 2006. Divulga o Pacto pela Saúde 2006. Consolidação do SUS e aprova as Diretrizes Operacionais do referido Pacto. [acesso 28 fev 2014]. Disponível em http://dtr2001. saude.gov.br/sas/PORTARIAS/port2006/GM/GM-399.htm

7. Brasil. Ministério da Saúde. Portaria GM n. 816, de 26 de julho de 2000. Aprova as diretrizes, destinadas a orientar, em todos os níveis do Sistema Único de Saúde, as medidas de prevenção, diagnóstico, tratamento e controle de hanseníase no país. Diário Oficial da União, Brasília, 27 jun. 2000; Seção 1, p. 26.

8. Brasil. Ministério da Saúde. Portaria GM n. 125, de 26 de março de 2009. Define as ações de controle da hanseníase. Diário Oficial da União, Brasília, 26 mar. 2009; Seção 1, p.73.

9. Fernandes DRF, Telles Filho PCP. Oficina de mobilização social em hanseníase: relato de experiência. Rev Bras Enferm. 2008; 61(supl.): 764-6.

10. Ayres JRCM, Calazans GJ, Saletti Filho HC, França Júnior I. Risco, vulnerabilidade e práticas de prevenção e promoção da saúde. In: Campos GWS, Minayo MCS, Akerman M, Drumond Junior M, Carvalho YM organizadores. Tratado de Saúde Coletiva. São Paulo: Hucitec, Rio de Janeiro: Fiocruz; 2006. p. 375-418.

11. Brasil. Ministério da Saúde. Secretaria de Atenção à Saúde. Departamento de Atenção Básica. Política Nacional de Atenção Básica. Brasília: Ministério da Saúde; 2006.

12. Brasil. Ministério da Saúde. Secretaria de Vigilância em Saúde. Departamento de Vigilância Epidemiológica. Manual de condutas para tratamento de úlceras em hanseníase e diabetes. Brasília: Ministério da Saúde; 2008.

13. Brasil. Ministério da Saúde. Portaria GM n. 198, de 13 de fevereiro de 2004. Institui a Política de Educação Permanente em Saúde como estratégia do SUS para a formação e desenvolvimento de trabalhadores para o setor e dá outras providências. Brasília; 2004.

14. Monroe AA, Gonzáles RIC, Palha PF, Sassaki CM, Ruffino Neto A, Vendramini SHF et al. Envolvimento de equipes da atenção básica à saúde no controle da tuberculose. Rev Esc Enferm USP. 2008; 42(2):262-7.

15. Peduzzi M, Guerra DAD, Braga CP, Lucena FS, Silva JAM. Atividades educativas de trabalhadores na atenção primária: concepções de educação permanente e de educação continuada em saúde presentes no cotidiano de Unidades Básicas de Saúde em São Paulo. Interface (Botucatu). 2009; 13(30):121-34.

16. Carneiro Junior N, Silveira C. Organização das práticas de atenção primária em saúde no contexto dos processos de exclusão/inclusão social. Cad Saúde Pública. 2003; 19(6):1827-35.

17. Sistema de Informação da Atenção Básica. Centro de Saúde Escola Barra Funda Dr. Alexandre Vranjac, 2013.

18. Oliveira WLW, Mendes CM, Tardin RT. Social representation of Hansen's disease thirty years after the term "leprosy" was replaced in Brazil. Hist. Cienc. Saúde-manguinhos. 2003; (10):41-8.

19. Silva JAS, Val LF, Nichiata LYI. A estratégia saúde da família e a vulnerabilidade programática na atenção ao HIV/AIDS: uma revisão da literatura. Mundo Saúde. 2010; 34(1):103-8.

20. Starfield, B. Atenção Primária: equilíbrio entre necessidades de saúde, serviços e tecnologia. Brasília: Unesco; 2002. 21. Nunes JM, Oliveira EM, Vieira NFC. Hanseníase: conhecimentos e mudanças na vida das pessoas acometidas. Ciên Saúde Colet. 2011; 16(Supl. 1):1311-18.

Recebido em 15 de dezembro de 2014. Aprovado em 09 de outubro de 2015. 